\title{
ELASTIC ANALYSIS OF AN AXISYMMETRIC STRESS FIELD PERTURBED BY A SPHEROIDAL INHOMOGENEITY*
}

\author{
BY \\ W. T. CHEN
}

IBM Systems Development Division, Endicott, New York

\begin{abstract}
An infinite elastic medium contains an elastic spheroidal inclusion. Both materials are transversely isotropic. Assuming that the stress field in the absence of any inhomogeneity is prescribed, it is desired to calculate the modification caused by the inclusion. This paper presents a general solution to this elasticity problem with the restriction that the prescribed stress field is axisymmetric. The analysis is based upon some new identities in Legendre functions, which are derived in this paper. The solution is in the form of combinations of Legendre functions. An example of a spheroidal cavity in a tension field is given.
\end{abstract}

1. Introduction. In many mechanical design problems, it is necessary to estimate the stresses in the neighborhood of inhomogeneities, cavities, or holes. Assuming that the stress field in the absence of any inhomogeneity is known, it is desired to calculate the modification caused by the inhomogeneity. Also, one may wish to find the stresses induced by a change in shape or size of the inhomogeneity due to a martensitic transformation or an uneven thermal expansion. Recent interest in such stress problems also stems from the development and application of composite materials in industry.

There are many exact solutions in linear elasticity theory which give a detailed knowledge of the stresses around inhomogeneities of simple mathematical shapes in media of infinite extent. A review of significant contributions in the area of threedimensional stress concentration is contained in a survey paper by Sternberg [1] in 1958. The earliest works on three-dimensional problems were concerned with spherical cavities in isotropic materials under homogeneous stresses at infinity. Later many investigators considered spheroidal and ellipsoidal shaped inhomogeneities, and in some instances the inhomogeneity materials were taken to be elastic, and both materials to be anisotropic. Since the publication of Sternberg's survey, Eshelby [2] has given a general review of the problem, with a particularly detailed account of his method of solution. Some recent contributions are cited by Chen [3] and by Sendeckyj [4]. In most of these works, the stress field perturbed by the inhomogeneity is homogeneous (tension, shear), or linear (bending and torsion). Recently, Podil'chuk [5] ${ }^{1}$ has considered the problem of a spheroidal cavity in an infinite isotropic medium subjected to prescribed axisymmetric torsionless surface traction, which may be of a general nature, on the cavity surface. Podil'chuk illustrates his analysis with the example of a spheroidal cavity in a radially

* Received November 10, 1969.

1 The author wishes to express his deep appreciation to Professor G. P. Sendeckyj for making available to the author an English translation of Podil'chuk's paper. 
symmetrical bending field. A knowledge of the results in [3] and [5] suggests that the solution to the problem of a spheroidal elastic inclusion contained in an infinite transversely isotropic medium under prescribed axisymmetric loading can be easily obtained, if the stress field in this medium without the inclusion is known. It turns out that the conjecture is true. The analytical results have a very simple form. Formally the isotropic solution can be obtained from the corresponding transversely isotropic one by a suitable limiting process. That this is not a trivial matter is evident from the work of Podil'chuk [5] for the case of a cavity (an infinitely weak inclusion).

2. Description and formulation of the physical problem. An elastic component is strained by external loads. The stress field induced by these forces has been obtained with the assumption that the material is elastic and homogeneous. How would this stress field be altered if there is an elastic inhomogeneity in the shape of a spheroid, situated inside the component? This question will be answered here using the linear elasticity theory, with the restriction that the stress field is axisymmetric. The inclusion and outside materials are assumed to both be transversely isotropic. In transversely isotropic materials, the physical property has an axis of symmetry. We shall assume that. this axis is parallel to the axis of the spheroid. At the interface, the traction and displacements are continuous. Since the main interest would be in the neighborhood of the inclusion, the outside material is taken to be infinite in extent. The shape of the spheroidal inclusion is defined by

$$
\frac{z^{2}}{a^{2}}+\frac{r^{2}}{b^{2}}=1
$$

where $a$ and $b$ are two semiaxes of the spheroid.

We shall call the stress field, which is obtained under the condition that the material is homogeneous, the 'unperturbed stress field'. In this paper, it will be assumed that this stress field is torsionless and axisymmetric. The surface traction associated with this unperturbed stress field (superscript $U$ ) is given by

$$
\begin{gathered}
\sigma_{r z}^{\prime \prime}+\sigma_{z=}^{\prime \prime}\left(n_{z} / n_{r}\right)=(a / r)\left[D_{0}+\sum_{n=1}^{\infty}(2 n+1) D_{n} P_{n}(z / a)\right], \\
\sigma_{r r}^{\prime \prime}+\sigma_{r z}^{\prime \prime}\left(n_{z} / n_{r}\right)=\sum_{n=1}^{\infty}\left({ }^{\prime} n+1\right) E_{n}^{\prime} P_{n}^{\prime}(z / a) .
\end{gathered}
$$

$D_{n}$ and $E_{n}$ are known constants. $P_{n}$ is the Legendre polynomial of the $n$th degree; $n_{r}$ and $n_{z}$ are the direction cosines of the normal vector to the spheroid. This unperturbed stress distribution must be self-equilibrating, i.e.,

$$
\int_{-a}^{a}\left[\sigma_{r z}^{\ell}+\sigma_{z z}^{U}\left(n_{z} / n_{r}\right)\right] r d z=0 .
$$

It follows that in Eq. (2) the constant

$$
D_{0}=0 \text {. }
$$

The problem now is to find the stress field inside the inclusion, and an additional stress field for the outside medium to be superimposed upon the unperturbed stress field, so that traction and displacements are continuous across the interface. These continuity conditions are: 


$$
\begin{gathered}
\left(\sigma_{z z}-\sigma_{z z}^{I}\right) n_{z}+\left(\sigma_{r z}-\sigma_{r z}^{I}\right) n_{r}=0, \\
\left(\sigma_{r r}-\sigma_{r r}^{I}\right) n_{r}+\left(\sigma_{r z}-\sigma_{r z}^{I}\right) n_{z}=0, \\
u_{r}=u_{r}^{I}, \quad u_{z}=u_{z}^{I} .
\end{gathered}
$$

The superscript $I$ designates quantities relating to the inclusion.

Finally, it is reasonable to expect that at a distance from the inclusion the perturbing effect of the inclusion on the stress field gradually disappears.

3. Potential functions solution. The method of analysis used in this paper is based upon the potential functions solution of the homogeneous displacement equations of equilibrium in a transversely isotropic elastic medium. A brief account of the method is given in the treatise by Green and Zerna [6]. We shall use a cylindrical coordinate system $(r, \theta, z)$, where the $z$-axis is parallel to the material axis of symmetry. We shall also introduce a set of dimensionless parameters $\nu_{\alpha}, k_{\alpha}(\alpha=1,2) . \nu_{1}$ and $\nu_{2}$ are roots of the equation

$$
c_{11} c_{44} \nu^{2}+\left[c_{13}\left(2 c_{44}+c_{13}\right)-c_{11} c_{13}\right] \nu+c_{33} c_{44}=0 .
$$

$k_{1}$ and $k_{2}$ are defined by

$$
k_{\alpha}=\left(c_{11} \nu_{\alpha}-c_{44}\right) /\left(c_{13}+c_{44}\right), \quad(\alpha=1,2) .
$$

It has been found convenient to employ two new variables

$$
z_{\alpha}=z / \sqrt{ } \nu_{\alpha}, \quad(\alpha=1,2) .
$$

A set of displacement and stresses which satisfy the equilibrium equations may be derived from two potential functions $\phi_{1}\left(r, z_{1}\right)$ and $\phi_{2}\left(r, z_{2}\right)$, where $\phi_{1}(r, z)$ and $\phi_{2}\left(r, z_{2}\right)$ are harmonic in the $(r, \theta, z)$ space. These stresses and displacements are:

$$
\begin{aligned}
& c_{44} u_{r}=\frac{\partial}{\partial r}\left[\phi_{1}\left(r, z_{1}\right)+\phi_{2}\left(r, z_{2}\right)\right], \\
& c_{14} u_{z}=\frac{k_{1}}{\sqrt{\nu_{1}}} \frac{\partial \phi_{1}\left(r, z_{1}\right)}{\partial z_{1}}+\frac{k_{2}}{\sqrt{\nu_{2}}} \frac{\partial \phi_{2}\left(r, z_{2}\right)}{\partial z_{2}} . \\
& c_{44} u_{\theta}=0 \text {, } \\
& \sigma_{z 2}=\left(1+k_{1}\right) \frac{\partial^{2} \phi_{1}\left(r, z_{1}\right)}{\partial z_{1}^{2}}+\left(1+k_{2}\right) \frac{\partial^{2} \phi_{2}\left(r, z_{2}\right)}{\partial z_{2}} \\
& \sigma_{r_{2}}=\frac{1+k_{1}}{\sqrt{ } \nu_{1}} \frac{\partial^{2} \phi_{1}\left(r, z_{1}\right)}{\partial z_{1} \partial r}+\frac{1+k_{2}}{\sqrt{ } \nu_{2}} \frac{\partial^{2} \phi_{2}\left(r, z_{2}\right)}{\partial z_{2} \partial r} \\
& \sigma_{r r}=\frac{1+k_{1}}{\nu_{1}}\left(\frac{\partial^{2}}{\partial r^{2}}+\frac{\partial}{r \partial r}\right) \phi_{1}\left(r, z_{1}\right)+\frac{1+k_{2}}{\nu_{2}}\left(\frac{\partial^{2}}{\partial r^{2}}+\frac{\partial}{r \partial r}\right) \phi_{2}\left(r, z_{2}\right) \\
& -\frac{c_{11}-c_{12}}{c_{44}} \frac{1}{r}\left[\frac{\partial \phi_{1}\left(r, z_{1}\right)}{\partial r}+\frac{\partial \phi_{2}\left(r, z_{2}\right)}{\partial r}\right] \text {, } \\
& \sigma_{\theta \theta}=\frac{1+k_{1}}{\nu_{1}}\left(\frac{\partial^{2}}{\partial r^{2}}+\frac{1}{r} \frac{\partial}{\partial r}\right) \phi_{1}\left(r, z_{1}\right)+\frac{1+k_{2}}{\nu_{2}}\left(\frac{\partial^{2}}{\partial r^{2}}+\frac{1}{r} \frac{\partial}{\partial r}\right) \phi_{2}\left(r, z_{2}\right) \\
& -\frac{c_{11}-c_{12}}{c_{44}} \frac{\partial^{2}}{\partial r^{2}}\left[\phi_{1}\left(r, z_{1}\right)+\phi_{2}\left(r, z_{2}\right)\right] \text {. }
\end{aligned}
$$


In Eqs. (10) and (11) we have already limited the analysis to the axisymmetric torsionless situation.

4. Analysis. Define

$$
a_{\alpha}^{2}=a^{2} / \nu_{\alpha}, \quad c_{\alpha}^{2}=a_{\alpha}^{2}-b^{2}, \quad \alpha=1,2 .
$$

The spheroidal system $\left(\eta_{\alpha}, \phi_{\alpha}, \theta\right)$ will be employed. Its relation to the cylindrical coordinate system $\left(r, \theta, z_{\alpha}\right)$ is given by $(\alpha=1,2)$

$$
z_{\alpha}=c_{\alpha} q_{\alpha} p_{\alpha}, \quad r=c_{\alpha}\left(q_{\alpha}^{2}-1\right)^{1 / 2}\left(1-p_{\alpha}\right)^{1 / 2},
$$

where for $\operatorname{Re}\left[c_{\alpha}^{2}\right]>0$,

$$
q_{\alpha}=\cosh \eta_{\alpha}, \quad p_{\alpha}=\cos \phi_{\alpha}
$$

and for $\operatorname{Re}\left[c_{\alpha}^{2}\right]<0$,

$$
q_{\alpha}=i \sinh \eta_{\alpha}, \quad p_{\alpha}=\cos \phi_{\alpha} .
$$

In the latter case it is customary to have the imaginary part of $c_{\alpha}$ negative.

Note that when $q_{\alpha}^{2}=\rho_{\alpha}^{2} \equiv a_{\alpha}^{2} / c_{\alpha}^{2}$, Eq. (13) reduces to the spheroidal surface given by Eq. (1), and $p_{\alpha}=z / a$. We shall adopt the convention that quantities associated with the inclusion material have the superscript $I$, e.g.

$$
\left(a_{\alpha}^{I}\right)^{2}=a^{2} / \nu_{\alpha}^{I}, \quad\left(c_{\alpha}^{I}\right)^{2}=\left(a_{\alpha}^{I}\right)^{2}-b^{2} .
$$

The ratio of the direction cosines of the normal to the interface may be expressed in terms of $p_{\alpha}$ :

$$
\frac{n_{z}}{n_{r}}=\frac{p_{\alpha}}{\left(1-p_{\alpha}^{2}\right)^{1 / 2 / 2}} \frac{\left(\rho_{\alpha}^{2}-1\right)^{1 / 2}}{\rho_{\alpha} \sqrt{\nu_{\alpha}}}
$$

From Eqs. (10) and (11)

$$
\begin{array}{r}
\sigma_{r 2}+\sigma_{z z} \frac{n_{z}}{n_{r}}=\frac{1+k_{1}}{\sqrt{ } \nu_{1}}\left\{\frac{\partial^{2} \phi_{1}}{\partial r \partial z_{1}}+\sqrt{ } \nu_{1} \frac{\partial^{2} \phi_{1}}{\partial z_{1}^{2}} \frac{n_{z}}{n_{r}}\right\}+\frac{1+k_{2}}{\sqrt{ } \nu_{2}}\left\{\frac{\partial^{2} \phi_{2}}{\partial r \partial z_{2}}+\sqrt{ } \nu_{2} \frac{\partial^{2} \phi_{2}}{\partial z_{2}^{2}} \frac{n_{z}}{n_{r}}\right\} \\
\sigma_{r r}+\sigma_{r z} \frac{n_{z}}{n_{r}}=-\frac{1+k_{1}}{\sqrt{ } \nu_{1}}\left\{\frac{\partial^{2} \phi_{1}}{\partial z_{1}^{2}}-\sqrt{\nu_{1}} \frac{\partial^{2} \phi_{1}}{\partial r \partial z_{1}} \frac{n_{z}}{n_{r}}\right\}-\frac{1+k_{2}}{\nu_{2}}\left\{\frac{\partial^{2} \phi_{2}}{\partial z_{2}^{2}}-\sqrt{\nu_{2}} \frac{\partial^{2} \phi_{2}}{\partial r \partial z_{2}} \frac{n_{z}}{n_{r}}\right\} \\
-\frac{c_{11}-c_{12}}{c_{44}} \frac{1}{r}\left\{\frac{\partial \phi_{1}}{\partial r}+\frac{\partial \phi_{2}}{\partial r}\right\} .
\end{array}
$$

At the surface of the spheroidal cavity, the above expressions reduce to

$$
\begin{aligned}
\sigma_{r z}+\sigma_{z z} \frac{n_{z}}{n r}= & \frac{a}{r} \frac{b^{2}}{a^{2}}\left\{\left.\frac{1+k_{1}}{c_{1}} \frac{\partial^{2} \phi_{1}}{\partial q_{1} \partial z_{1}}\right|_{a_{1}-\rho_{1}}+\left.\frac{1+k_{2}}{c_{2}} \frac{\partial^{2} \phi_{2}}{\partial q_{2} \partial z_{2}}\right|_{a_{0}-\rho_{0}}\right\}, \\
\sigma_{r r}+\sigma_{r z} \frac{n_{2}}{n_{r}}= & -\frac{1}{a}\left\{\left.\frac{1+k_{1}}{\sqrt{ } \nu_{1}} \frac{\partial^{2} \phi_{1}}{\partial p_{1} \partial z_{1}}\right|_{a_{1}-\rho_{1}}+\left.\frac{1+k_{2}}{\sqrt{ } \nu_{2}} \frac{\partial^{2} \phi_{2}}{\partial p_{2} \partial z_{2}}\right|_{a_{2}-\rho_{0}}\right\} \\
& -\frac{c_{11}-c_{12}}{c_{44}} \frac{1}{r}\left\{\left.\frac{\partial \phi_{1}}{\partial r}\right|_{a_{1}-\rho_{2}}+\left.\frac{\partial \phi_{2}}{\partial r}\right|_{e_{2}-\rho_{0}}\right\}
\end{aligned}
$$

The potential functions $\phi_{1}\left(r, z_{1}\right)$ and $\phi_{2}\left(r, z_{2}\right)$ associated with the additional or per- 
turbing stress field are taken to be

$$
\phi_{\alpha}\left(r, z_{\alpha}\right)=\sum_{n=1}^{\infty} A_{\alpha n}\left[P_{n+1}\left(p_{\alpha}\right) Q_{n+1}\left(q_{\alpha}\right)-P_{n-1}\left(p_{\alpha}\right) Q_{n-1}\left(q_{\alpha}\right)\right]
$$

$P_{n}$ and $Q_{n}$ are Legendre functions of the first kind and second kind respectively. A property of the harmonic function representation in Eq. (20) is that the partial derivatives with respect to $r$ and $z_{\alpha}$ are in very simple forms. They are

$$
\begin{gathered}
\frac{\partial}{\partial r}\left[P_{n+1}\left(p_{\alpha}\right) Q_{n+1}\left(q_{\alpha}\right)-P_{n-1}\left(p_{\alpha}\right) Q_{n-1}\left(q_{\alpha}\right)\right]=-\frac{r(2 n+1) P_{n}^{\prime}\left(p_{\alpha}\right) Q_{n}^{\prime}\left(q_{\alpha}\right)}{n(n+1) c_{\alpha}^{2}}, \\
\frac{\partial}{\partial z_{\alpha}}\left[P_{n+1}\left(p_{\alpha}\right) Q_{n+1}\left(q_{\alpha}\right)-P_{n-1}\left(p_{\alpha}\right) Q_{n-1}\left(q_{\alpha}\right)\right]=\frac{2 n+1}{c_{\alpha}} P_{n}\left(p_{\alpha}\right) Q_{n}\left(q_{\alpha}\right) .
\end{gathered}
$$

The above relations are also valid if $Q_{n+1}\left(q_{\alpha}\right), Q_{n}\left(q_{\alpha}\right), Q_{n-1}\left(q_{\alpha}\right)$, are replaced by $P_{n+1}\left(q_{\alpha}\right), P_{n}\left(q_{\alpha}\right)$, and $P_{n-1}\left(q_{\alpha}\right)$ respectively. (See Appendix.)

Combining Eqs. (18) to (22), the traction and displacement at the spheroidal interface (i.e., $q_{a}=\rho_{\alpha}$ ) are

$$
\begin{gathered}
\sigma_{r z}+\sigma_{z z} \frac{n_{z}}{n_{r}}=\left(\frac{a}{r}\right) \frac{b^{2}}{a^{2}} \sum_{n=1}^{\infty} \sum_{\alpha=1}^{2} \frac{A_{\alpha n}(2 n+1)\left(1+k_{\alpha}\right)}{c_{\alpha}^{2}} Q_{n}^{\prime}\left(\rho_{\alpha}\right) P_{n}(z / a), \\
\sigma_{r r}+\sigma_{r s} \frac{n_{z}}{n_{r}}=-\sum_{n=1}^{\infty} \sum_{\alpha=1}^{2} A_{\alpha n}(2 n+1)\left[\frac{1+k_{\alpha}}{a c_{\alpha} \sqrt{\nu_{\alpha}}} Q_{n}\left(\rho_{\alpha}\right)-\frac{c_{11}-c_{12}}{c_{44} c_{\alpha}^{2} n(n+1)} Q_{n}^{\prime}\left(\rho_{\alpha}\right)\right] P_{n}^{\prime}(z / a), \\
c_{44} u_{r}=-r \sum_{n=1}^{\infty} \sum_{\alpha=1}^{2} \frac{A_{\alpha n}(2 n+1)}{n(n+1) c_{\alpha}^{2}} Q_{n}^{\prime}\left(\rho_{\alpha}\right) P_{n}^{\prime}(z / a), \\
c_{44} u_{z}=\sum_{n=1}^{\infty} \sum_{\alpha=1}^{2} \frac{A_{\alpha n}(2 n+1) k_{\alpha}}{c_{\alpha} \sqrt{ } \nu_{\alpha}} Q_{n}\left(\rho_{\alpha}\right) P_{n}(z / a) .
\end{gathered}
$$

The foregoing results from Eqs. (23) to (26) can also be derived if the Legendre function $Q_{n}\left(\rho_{\alpha}\right)$ is replaced by $P_{n}\left(\rho_{\alpha}\right)$. An examination of Eqs. (2) and (3) leads one to conclude that each of the potential functions of the unperturbed stress field in the vicinity of the inhomogeneity can be expressed in the form

$$
\phi_{\alpha}^{U}\left(r, z_{\alpha}\right)=\sum_{n=1}^{\infty} C_{\alpha n}\left[P_{n+1}\left(p_{\alpha}\right) P_{n+1}\left(q_{\alpha}\right)-P_{n-1}\left(p_{\alpha}\right) P_{n-1}\left(q_{\alpha}\right)\right]
$$

Each pair of the constants $C_{1 n}, C_{2 n}$ is determined from the equations

$$
\begin{gathered}
\frac{b^{2}}{a^{2}} \sum_{\alpha=1}^{2} \frac{\left(1+k_{\alpha}\right) P_{n}^{\prime}\left(\rho_{\alpha}\right)}{c_{\alpha}^{2}} C_{\alpha n}=D_{n} \\
-\sum_{\alpha=1}^{2}\left[\frac{\left(1+k_{\alpha}\right) P_{n}\left(\rho_{\alpha}\right)}{\sqrt{ } \nu_{\alpha} c_{\alpha} a}-\frac{\left(c_{11}-c_{12}\right) P_{n}^{\prime}\left(\rho_{\alpha}\right)}{c_{44} c_{\alpha}^{2} n(n+1)}\right] C_{\alpha n}=E_{n} .
\end{gathered}
$$

Eqs. (27) to (29) provide a convenient way to find the 'unperturbed' displacement field from a knowledge of the surface traction of the unperturbed field at the interface. In some problems, one may find that this displacement field can be more easily written down without going through this procedure.

Note that Eq. (27) does not contain any singularity inside of the inclusion. This is also a requirement of the potential functions for the inclusion medium. These potential 
functions will assume the forms

$$
\phi_{\alpha}^{I}\left(r, z_{\alpha}^{I}\right)=\sum_{n=1}^{\infty} \sum_{\alpha=1}^{2} B_{\alpha n}\left[P_{n+1}\left(p_{\alpha}^{I}\right) P_{n+1}\left(q_{\alpha}^{I}\right)-P_{n-1}\left(p_{\alpha}^{I}\right) P_{n-1}\left(q_{\alpha}^{I}\right)\right]
$$

The superscript $I$ indicates that the quantities $\phi_{\alpha}^{I}, p_{\alpha}^{I}, q_{\alpha}^{I}$, etc., are associated with the inclusion. The constants $A_{1 n}, A_{2 n}, B_{1 n}, B_{2 n}(n=1 \cdots \infty)$ are determined from the four continuity conditions specified by Eq. (6).

The four algebraic equations resulting from the continuity conditions are:

$$
\begin{aligned}
& \sum_{\alpha=1}^{2}\left[\frac{\left(1+k_{\alpha}\right) A_{\alpha n} Q_{n}^{\prime}\left(\rho_{\alpha}\right)}{c_{\alpha}^{2}}-\frac{\left(1+k_{\alpha}^{I}\right) B_{\alpha n} P_{n}^{\prime}\left(\rho_{\alpha}^{I}\right)}{\left(c_{\alpha}^{\mathrm{I}}\right)^{2}}\right]=-\frac{a^{2} D_{n}}{b^{2}}, \\
& \sum_{\alpha=1}^{2}\left[\frac{\left(1+k_{\alpha}\right) A_{\alpha n} Q_{n}\left(\rho_{\alpha}\right)}{c_{\alpha} \sqrt{\nu_{\alpha} a}}-\frac{\left(c_{11}-c_{12}\right) A_{\alpha n} Q_{n}^{\prime}\left(\rho_{\alpha}\right)}{c_{44} c_{\alpha}^{2} n(n+1)}-\frac{\left(1+k_{\alpha}^{I}\right) B_{\alpha n} P_{n}\left(\rho_{\alpha}^{I}\right)}{c_{\alpha} \sqrt{ } \nu_{\alpha}^{I} a}\right. \\
& \left.+\frac{\left(c_{11}^{I}-c_{12}^{I}\right) B_{\alpha n} P_{n}^{\prime}\left(\rho_{\alpha}^{I}\right)}{c_{44}^{\mathrm{I}}\left(c_{\alpha}^{\mathrm{I}}\right)^{2} n(n+1)}\right]=E_{n} \\
& \sum_{\alpha=1}^{2}\left[\frac{A_{\alpha n} Q_{n}^{\prime}\left(p_{\alpha}\right)+C_{\alpha n} P_{n}^{\prime}\left(\rho_{\alpha}\right)}{c_{44} c_{\alpha}^{2}}-\frac{B_{\alpha n} P_{n}^{\prime}\left(\rho_{\alpha}^{I}\right)}{c_{44}^{I}\left(c_{\alpha}^{I}\right)^{2}}\right]=0 \\
& \sum_{\alpha=1}^{2}\left[\frac{k_{\alpha}\left[A_{\alpha n} Q_{n}\left(\rho_{\alpha}\right) \pm C_{\alpha n} P_{n}\left(\rho_{\alpha}\right)\right]}{c_{44} \sqrt{ } \nu_{\alpha} c_{\alpha}}-\frac{k_{\alpha}^{I} B_{\alpha n} P_{n}\left(\rho_{\alpha}^{I}\right)}{c_{44}^{\mathrm{I}} \sqrt{ } \eta_{\alpha}^{\mathrm{I}} c_{\alpha}^{\mathrm{I}}}\right]=0 .
\end{aligned}
$$

The constants $C_{1 n}$ and $C_{2 n}$ in Eqs. (33) and (34) have already been determined by Eqs. (28) and (29). For each integer $n(n=1 \cdots \infty)$, there are four equations uniquely to determine the four constants $A_{\alpha_{n}}, B_{\alpha n}(\alpha=1,2)$.

The solution to the elasticity problem defined by Eqs. (1), (2), (3) and (6) are now at hand. They are given in terms of the potential functions specified by Eqs. (20), (27) and (30). The constants associated with these potential functions are uniquely determined by Eqs. (28), (29), (31), (32), (33) and (34).

In the case of a spheroidal cavity (very weak inclusion) the constants $B_{\alpha n}$ 's may be set equal to zero, and the constants $A_{\alpha n}$ 's are determined by Eqs. (31) and (32).

Example. Suppose the unperturbed stress field is a homogeneous one given by

$$
\sigma_{z z}^{U}=t_{z}, \quad \sigma_{r r}^{U}=t_{r}
$$

and all other stress components vanish. From Eqs. (2) and (3),

$$
D_{1}=t_{z} b^{2} / 3 a, \quad E_{1}=t_{r} / 3 \text {. }
$$

The potential functions $\phi_{1}$ and $\phi_{2}$ of the additional stress field are given in Eq. (20)

$$
\phi_{\alpha}\left(r, z_{\alpha}\right)=A_{\alpha 1}\left[P_{2}\left(p_{\alpha}\right) Q_{2}\left(q_{\alpha}\right)-Q_{0}\left(q_{\alpha}\right)\right], \quad \alpha=1,2 .
$$

The constants $A_{11}$ and $A_{12}$ are determined by the equations

$$
\frac{\left(1+k_{1}\right) Q_{1}^{\prime}\left(\rho_{1}\right) A_{11}}{c_{1}^{2}}+\frac{\left(1+k_{2}\right) Q_{2}^{\prime}\left(\rho_{2}\right) A_{12}}{c_{2}^{2}}=-\frac{t_{2}}{3},
$$




$$
\begin{aligned}
& {\left[\frac{\left(1+k_{1}\right) Q_{1}\left(\rho_{1}\right)}{\nu_{1} \rho_{1} c_{1}^{2}}-\frac{\left(c_{11}-c_{12}\right) Q_{1}^{\prime}\left(\rho_{1}\right)}{c_{44} c_{1}^{2}}\right] A_{11}} \\
& +\left[\frac{\left(1+k_{2}\right) Q_{1}\left(\rho_{2}\right)}{\nu_{2} \rho_{2} c_{2}^{2}}-\frac{\left(c_{11}-c_{12}\right)}{c_{44} c_{2}^{2}} Q_{1}^{\prime}\left(\rho_{2}\right)\right] A_{12}=\frac{t_{r}}{3} .
\end{aligned}
$$

This solution is identical to the results given in an earlier paper by Chen [3].

5. Discussion. An exact analysis, in the realm of linear elasticity, has been presented to account for the effect of a spheroidal elastic inhomogeneity embedded in an otherwise homogeneous medium. Both materials are transversely isotropic. The 'unperturbed' stress field is assumed to be axisymmetric. In the analysis, it is assumed that the surface tractions at the interface due to this unperturbed stress field are prescribed. The same analysis can, of course, be carried through if the displacements corresponding to the unperturbed stress field are prescribed, instead of the surface tractions. This analysis is also applicable to the situation when the stress field is induced by a prescribed axisymmetric inelastic strain inside the spheroidal inclusion. It may be shown that the induced strain field is a polynomial of degree $2 n$ in $r$ and $z$, when the prescribed inelastic strain is a polynomial of degree $2 n$ in $r$ and $z$. Similar conclusions have been reached by Eshelby [2] and Sendeckyj [4] for the isotropic ellipsoidal inclusion problem under more general loading conditions.

Appendix. Let a spheroidal coordinate system $(\eta, \phi, \theta)$, and a cylindrical coordinate system $(r, \theta, z)$ be related by

$$
z=c q p, \quad r=c\left(q^{2}-1\right)^{1 / 2}\left(1-p^{2}\right)^{1 / 2},
$$

where for $\operatorname{Re}\left[c^{2}\right]>0$, (prolate spheroidal system)

$$
q=\cosh \eta, \quad p=\cos \phi,
$$

and for $\operatorname{Re}\left[c^{2}\right]<0$, (oblate spheroidal system)

$$
q=i \sinh \eta, \quad p=\sin \phi .
$$

The constant $c^{2}$ is a prescribed length parameter of the spheroidal system. It is specified that if $\operatorname{Re}\left[c^{2}\right]<0$, then $c$ is in the third quadrant.

One wishes to show that

$$
\begin{gathered}
\frac{\partial}{\partial z}\left[P_{n+1}(p) P_{n+1}(q)-P_{n-1}(p) P_{n-1}(q)\right]=\frac{2 n+1}{c} P_{n}(p) P_{n}(q), \\
\frac{\partial}{\partial r}\left[P_{n+1}(p) P_{n+1}(q)-P_{n-1}(p) P_{n-1}(q)\right]=-\frac{(2 n+1) r}{n(n+1) c^{2}} P_{n}^{\prime}(\rho) P_{n}^{\prime}(q) ;
\end{gathered}
$$

and

$$
\begin{gathered}
\frac{\partial}{\partial z}\left[P_{n+1}(p) Q_{n+1}(q)-P_{n-1}(p) Q_{n-1}(q)\right]=\frac{2 n+1}{c} P_{n}(p) Q_{n}(q), \\
\frac{\partial}{\partial r}\left[P_{n+1}(p) Q_{n+1}(q)-P_{n+1}(p) Q_{n-1}(q)\right]=\frac{(2 n+1) r}{n(n+1) c^{2}} P_{n}^{\prime}(\rho) Q_{n}^{\prime}(q) .
\end{gathered}
$$

A convenient way to prove the above pairs of formulas is to use the addition theorem for Legendre functions [7, p, 364-384]. Based upon the addition theorem for Legendre function of the first kind, one may write 


$$
P_{n}((z-i r \cos \theta) / c)=P_{n}(q) P_{n}(p)+2 \sum_{m=1}^{\infty}(-1)^{m} P_{n}^{m}(q) P_{n}^{-m}(p) \cos m \theta .
$$

Eq. (A5) leads to the identity (also see [7, p. 412])

$$
\begin{aligned}
& P_{n+1}(q) P_{n+1}(p)-P_{n-1}(q) P_{n-1}(p) \\
& =\frac{1}{2 \pi} \int_{-\pi}^{\pi}\left[P_{n+1}\left(\frac{z-i r \cos \theta}{c}\right)-P_{n-1}\left(\frac{z-i r \cos \theta}{c}\right)\right] d \theta .
\end{aligned}
$$

Differentiate both sides with respect to $z$ :

$$
\begin{aligned}
\frac{\partial}{\partial z}\left[P_{n+1}(q) P_{n+1}(p)-P_{n-1}(q) P_{n-1}(p)\right] & =\frac{1}{2 \pi} \int_{-\pi}^{\pi} \frac{2 n+1}{c} P_{n}\left(\frac{z-i r \cos \theta}{c}\right) d \theta \\
& =\frac{2 n+1}{c} P_{n}(q) P_{n}(p) .
\end{aligned}
$$

Differentiate both sides of Eq. (A6) with respect to $r$ :

$$
\begin{aligned}
\frac{\partial}{\partial r}\left[P_{n+1}(q) P_{n+1}(p)-P_{n-1}(q) P_{n-1}(p)\right] & =\frac{1}{2 \pi} \int_{-\pi}^{\pi} \frac{-i(2 n+1)}{c} P_{n}\left(\frac{z-i r \cos \theta}{c}\right) \cos \theta d \theta \\
& =\frac{i(2 n+1)}{c} P_{n}^{1}(q) P_{n}^{-1}(p) \\
& =-\frac{(2 n+1) r}{n(n+1) c^{2}} P_{n}^{\prime}(q) P_{n}^{\prime}(p) .
\end{aligned}
$$

This completes the proof for Eqs. (A1) and (A2). Note that the index $n$ is unrestricted.

The addition theorem for Legendre functions of the second kind enables one to write

$$
Q_{n}\left(\frac{z-i r \cos \theta}{c}\right)=P_{n}(p) Q_{n}(q)+2 \sum_{m=1}^{\infty}(-1)^{m} P_{n}^{-m}(p) Q_{n}^{m}(q) \cos m \theta .
$$

The pair of relations (A3) and (A4) may now be proved in exactly the same manner as for the first pair. For some ranges of values of $(z-i r \cos \theta) / c$, the addition theorem for $Q_{n}$ takes a different form. However, the end result for Eqs. (A3) and (A4) remains the same. There is the restriction that the index $n$ cannot be equal to -1 or zero.

It may also be shown that Eqs. (A1), (A2), (A3), and (A4) are special cases of the following more general formulas:

$$
\frac{\partial}{\partial z}\left[P_{n+1}^{m}(q) P_{n+1}^{-m}(p)-P_{n-1}^{m}(q) P_{n-1}^{-m}(p)\right]=\frac{2 n+1}{c} P_{n}^{m}(q) P_{n}^{-m}(p),
$$

and (a) $m \neq 1$,

$$
\begin{aligned}
\frac{\partial}{\partial r}\left[P_{n+1}^{m}(q) P_{n+1}^{-m}(p)-P_{n-1}^{m}(q) P_{n-1}^{-m}(p)\right] & \\
= & \frac{(2 n+1) i}{2 c}\left[P_{n}^{m+1}(q) P_{n}^{-(m+1)}(p)+p_{n}^{m-1}(q) P_{n}^{-(m-1)}(p)\right],
\end{aligned}
$$

(b) $m=1$,

$$
\frac{\partial}{\partial r}\left[P_{n+1}^{1}(q) P_{n+1}^{-1}(p)-P_{n-1}^{1}(q) P_{n-1}^{-1}(p)\right]=\frac{(2 n+1) i}{2 c}\left\{P_{n}(q) P_{n}(p)+P_{n}^{2}(q) P_{n}^{-2}(p)\right\}
$$


As before the terms $P_{n+1}^{m}(q)$, etc., can be replaced by terms of the form $Q_{n+1}^{m}(q)$. When $m=0$, Eqs. (A8) and (A9) reduce to Eqs. (A1) and (A2) respectively. The index $m$ must always be an integer. These formulas are useful in problems involving nonaxisymmetric loadings.

\section{REFERENCES}

[1] E. Sternberg, Three-dimensional stress concentrations in the theory of elasticity, Applied Mechanics Reviews, vol. 11, 1958, pp. 1-4

[2] J. D. Eshelby, Elastic inclusions and inhomogeneities, Progress in Solid Mechanics, vol. 2, NorthHolland, Amsterdam, 1961, pp. 87-14C

[3] W. T. Chen, Axisymmetric stress distribution around spheroidal inclusions and cavities in a transversely isotropic material, J. Appl. Mech. 35, Trans. ASME E 90, 770-773 (1968)

[4] G. P. Sendeckyj, Ellipsoidal inhomogeneity problem, Ph.D. dissertation, Northwestern University, 1967 (University Microfilms Order No. 67-15, 337)

[5] Yu. M. Podil'chuk, Deformation of an axisymmetrically loaded elastic spheroid, Prikl. Mat. Meh. 29, 85-91 (1965)

[6] A. E. Green and W. Zerna, Theoretical elasticity, Clarendon Press, Oxford, 1954, p. 180

[7] E. W. Hobson, The theory of spherical and ellipsoidal harmonics, Chelsea, New York, 1955 